\title{
Study of the $f_{2}(1270)$ and $a_{2}(1320)$ resonances in $\gamma^{*}\left(Q^{2}\right) \gamma$ collisions
}

\author{
N. N. Achasov, A. V. Kiselev, and G. N. Shestakov \\ Laboratory of Theoretical Physics, S. L. Sobolev Institute for Mathematics, 630090 Novosibirsk, Russia
}

\begin{abstract}
We discuss studies of the $Q^{2}$ dependence of the $f_{2}(1270)$ and $a_{2}(1320)$ production cross sections in $\gamma^{*}\left(Q^{2}\right) \gamma$ collisions at current and coming into operation colliders with a high luminosity. Changing the dominant helicity amplitude occurs in the reactions $\gamma^{*}\left(Q^{2}\right) \gamma \rightarrow f_{2}(1270)$ and $\gamma^{*}\left(Q^{2}\right) \gamma \rightarrow a_{2}(1320)$ with increasing $Q^{2}$. This is caused by the coming of the QCD asymptotics. It is shown that the transition to the asymptotic behavior of QCD in the amplitudes $\gamma^{*}\left(Q^{2}\right) \gamma \rightarrow f_{2}(1270), a_{2}(1320)$ is provided by the compensation of the contributions of ground vector states $\rho$ and $\omega$ in $Q^{2}$-channel with the contributions of their radial excitations.
\end{abstract}

PACS numbers: 13.40.Gp, 13.60.Le, 13.66.Jn

Physics of two-photons collisions entering into the era of ultra-high statistics gives unique opportunities to study the internal (quark-gluon) structure of hadrons [14]. For example, the recent experiments of the Belle Collaboration on the reactions $\gamma \gamma \rightarrow \pi^{+} \pi^{-}[5,6], \gamma \gamma \rightarrow \pi^{0} \pi^{0}$ [7], and $\gamma \gamma \rightarrow \pi^{0} \eta$ [8] established conclusively the smallness of the two-photon widths of the $f_{0}(980)$ and $a_{0}(980)$ resonances, which testifies in favor of their four-quark structure 9 11]. ${ }^{1}$

The measurements of the two-photon widths of the light pseudoscalar mesons $P=\pi^{0}, \eta, \eta^{\prime}$ in $\gamma \gamma$ collisions 1] and the transition form factors $F_{\gamma^{*} \gamma \rightarrow P}\left(Q^{2}\right)$ in $\gamma^{*}\left(Q^{2}\right) \gamma$ collisions ${ }^{2}$ performed by CELLO [12], CLEO [13], BaBar [14, 15] and Belle [16] Collaborations allowed to realize a critical test of QCD calculations of the processes at large $Q^{2}$.

Production of classical tensor $q \bar{q}$ resonances by two real photons proceeds very intensively: $f_{2}(1270)$ in the reactions $\gamma \gamma \rightarrow \pi^{+} \pi^{-}$[5, 6, 17, 18] and $\gamma \gamma \rightarrow \pi^{0} \pi^{0}$ 7, 19. and $a_{2}(1320)$ in the reactions $\gamma \gamma \rightarrow \pi^{0} \eta$ [8, 20] and $\gamma \gamma \rightarrow \pi^{+} \pi^{-} \pi^{0}$ [21 25] (see Fig. 1). This fact is a good reason to start detailed investigations of the $Q^{2}$ dependence of the $f_{2}(1270)$ and $a_{2}(1320)$ production cross sections in $\gamma^{*} \gamma$ collisions at $e^{+} e^{-}$colliders with a high luminosity. ${ }^{3}$

We now turn to the detailed discussion.

In $\gamma \gamma$ collisions, the $f_{2}(1270)$ and $a_{2}(1320)$ resonances can be produced in the states with helicity $\lambda=0$ and \pm 2 . Helicity $\lambda$ is defined in the resonance rest frame, in

${ }^{1}$ In 1982 , the prediction $\Gamma\left(f_{0}(980) \rightarrow \gamma \gamma\right) \approx \Gamma\left(a_{0}(980) \rightarrow \gamma \gamma\right) \approx$ $0.27 \mathrm{keV}$ was done in the four-quark MIT bag model [9, 10]. In 2014, the Particle Data Group cited in the Review of Particle Physics the following data [1]: $\Gamma\left(f_{0}(980) \rightarrow \gamma \gamma\right) \approx 0.29$ $\mathrm{keV}$ and $\Gamma\left(a_{0}(980) \rightarrow \gamma \gamma\right) \approx 0.3 \mathrm{keV}$, which is an order of magnitude smaller than the $\gamma \gamma$ width of the tensor $q \bar{q}$ meson $\Gamma\left(f_{2}(1270) \rightarrow \gamma \gamma\right) \approx 3 \mathrm{keV}$. The prediction of the $q \bar{q}$ model $\Gamma\left(f_{0}(980) \rightarrow \gamma \gamma\right) / \Gamma\left(a_{0}(980) \rightarrow \gamma \gamma\right)=25 / 9$ is excluded experimentally.

${ }^{2} \gamma^{*}\left(Q^{2}\right)\left(\gamma^{*}\right.$ below $)$ denotes the photon with virtuality $-Q^{2}$.

${ }^{3}$ Currently, the maximum luminosity $\approx 2 \cdot 10^{34} \mathrm{~cm}^{-2} \cdot c^{-1}$ is reached at the KEKB $e^{+} e^{-}$collider [1, 4]. The luminosity of $8 \cdot 10^{35} \mathrm{~cm}^{-2} \cdot c^{-1}$ is planned to have at the SuperKEKB factory [1, 3].
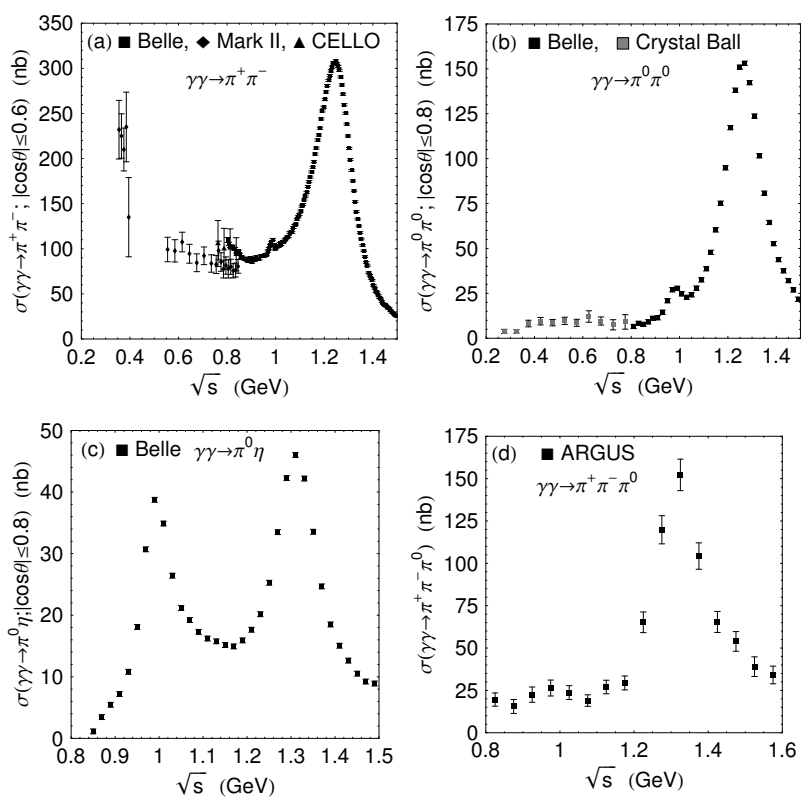

Figure 1: Cross sections of the reactions (a) $\gamma \gamma \rightarrow \pi^{+} \pi^{-}$ [5, 6, 17, 18], (b) $\gamma \gamma \rightarrow \pi^{0} \pi^{0}$ [7, 19], (c) $\gamma \gamma \rightarrow \pi^{0} \eta$ [8], and (d) $\gamma \gamma \rightarrow \pi^{+} \pi^{-} \pi^{0}$ [23] as functions of the invariant mass, $\sqrt{s}$, of the final meson system. In plots (a)-(c) $\theta$ denotes the polar angle of one of the outgoing mesons with respect to the incident photon direction in the $\gamma \gamma$ center-of-mass system. The reactions $\gamma \gamma \rightarrow \pi^{0} \pi^{0}$, plot (b), and $\gamma \gamma \rightarrow \pi^{+} \pi^{-} \pi^{0}$, plot (d), seem more preferable in the sense of the smallness of the physical background under the $f_{2}(1270)$ and $a_{2}(1320)$ peaks, respectively.

which $\lambda=\lambda_{1}-\lambda_{2}$, where $\lambda_{1}$ and $\lambda_{2}$ are the helicities of incoming photons. According to the high-statistics measurements $[6-8,17-19,23-25]$ the fraction of the $f_{2}(1270)$ and $a_{2}(1320)$ production in states with $\lambda= \pm 2$ in $\gamma \gamma$ collisions is more than $95 \%$.

This remarkable experimental fact of $\lambda= \pm 2$ dominance is naturally reproduced by the effective gaugeinvariant Lagrangian, describing the tensor meson production by two photons with opposite helicities only 
[10, 26, 27],

$$
L=g_{T \gamma \gamma} T_{\mu \nu} F_{\mu \sigma} F_{\nu \sigma}
$$

where $F_{\mu \nu}=\partial_{\mu} A_{\nu}-\partial_{\nu} A_{\mu}$ is the tensor of the electromagnetic field $A_{\mu}, T_{\mu \nu}$ is the field of the tensor meson $T\left(T=f_{2}(1270), a_{2}(1320)\right) ; T_{\mu \nu}=T_{\nu \mu}, T_{\mu \mu}=0$, $\partial_{\mu} T_{\mu \nu}=0 ; g_{T \gamma \gamma}$ is the coupling constants of the $T$ meson to the energy-momentum tensor of the electromagnetic field.

Using Lagrangian (1) one can unambiguously predict the hierarchy of the $Q^{2}$ dependencies of the helicity amplitudes $V_{\lambda_{1}, \lambda_{2}}^{(\lambda)}\left(T ; s, Q^{2}\right)=V_{-\lambda_{1},-\lambda_{2}}^{(-\lambda)}\left(T ; s, Q^{2}\right)$ describing the $\gamma^{*} \gamma \rightarrow T$ vertices [26, 28]:

$$
\begin{gathered}
V_{1,-1}^{(2)}\left(T ; s, Q^{2}\right)=V_{T}\left(s, Q^{2}\right)\left(1+\frac{Q^{2}}{s}\right), \\
V_{1,0}^{(1)}\left(T ; s, Q^{2}\right)=V_{T}\left(s, Q^{2}\right) \sqrt{\frac{Q^{2}}{2 s}}\left(1+\frac{Q^{2}}{s}\right), \\
V_{1,1}^{(0)}\left(T ; s, Q^{2}\right)=-V_{T}\left(s, Q^{2}\right) \frac{Q^{2}}{\sqrt{6} s}\left(1+\frac{Q^{2}}{s}\right) .
\end{gathered}
$$

Here $s=\left(q_{1}+q_{2}\right)^{2} ; q_{1}$ and $q_{2}$ are the four-momenta of the incident photons, $q_{1}^{2}=0, q_{2}^{2}=-Q^{2}$;

$$
\begin{gathered}
V_{T}\left(s, Q^{2}\right)=g_{T \gamma \gamma} s F_{T}\left(Q^{2}\right) / 2, \quad F_{T}(0)=1, \\
g_{T \gamma \gamma} s=2 V_{1,-1}^{(2)}(T ; s, 0)=\sqrt{320 \pi \sqrt{s} \Gamma_{T \rightarrow \gamma \gamma}(s)},
\end{gathered}
$$

and $F_{T}\left(Q^{2}\right)$ is the transition form factor which is common for all vertices.

The vertex $V_{1,0}^{(1)}\left(T ; s, Q^{2}\right)$ vanishes for $Q^{2} \rightarrow 0$ as $\sqrt{Q^{2}}$. This is a consequence of gauge invariance. The vertex $V_{1,1}^{(0)}\left(T ; s, Q^{2}\right)$ is proportional to $Q^{2}$ for $Q^{2} \rightarrow 0$ owing to a specific selection of the $\gamma^{*} \gamma T$ interaction which consists with the experimental fact of $\lambda= \pm 2$ dominance in $\gamma \gamma \rightarrow$ $T$ transitions (see $V_{1,-1}^{(2)}(T ; s, 0)$ in Eq. (6) ).

For small $Q^{2}$, the dominance of $V_{1,-1}^{(2)}\left(T ; s, Q^{2}\right)$ over $V_{1,0}^{(1)}\left(T ; s, Q^{2}\right)$ and $V_{1,1}^{(0)}\left(T ; s, Q^{2}\right)$ is certainly maintained. However, for large $Q^{2}$ the situation changes radically. Asymptotically

$$
\begin{gathered}
V_{1,-1}^{(2)}\left(T ; s, Q^{2}\right) \sim F_{T}\left(Q^{2}\right) Q^{2}, \\
V_{1,0}^{(1)}\left(T ; s, Q^{2}\right) \sim F_{T}\left(Q^{2}\right) Q^{3}, \\
V_{1,1}^{(0)}\left(T ; s, Q^{2}\right) \sim F_{T}\left(Q^{2}\right) Q^{4}
\end{gathered}
$$

and the $\gamma^{*} \gamma \rightarrow T$ vertex with $\lambda=0$ becomes dominant.

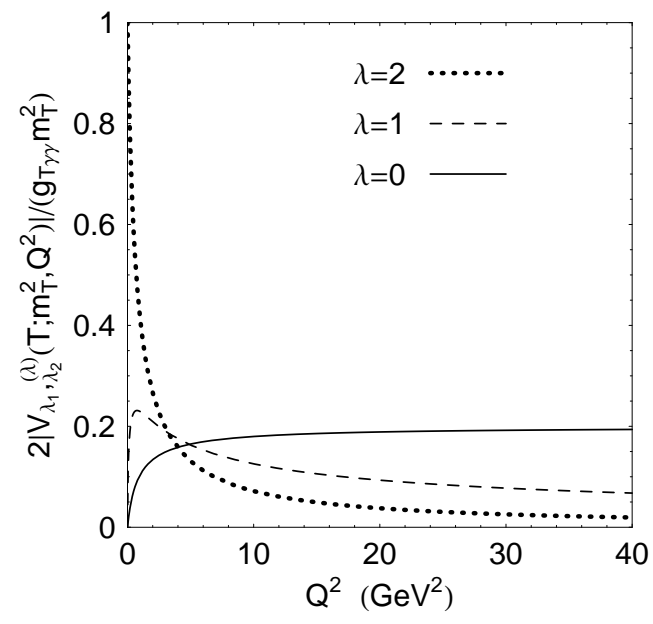

Figure 2: The $Q^{2}$ dependencies of the normalized vertex functions $2\left|V_{\lambda_{1}, \lambda_{2}}^{(\lambda)}\left(T ; m_{T}^{2}, Q^{2}\right)\right| /\left(g_{T \gamma \gamma} m_{T}^{2}\right)$ calculated according to Eqs. (2) $-(6)$ and (10). For the $f_{2}(1270)$ and $a_{2}(1320)$ resonances these dependencies practically coincide because $m_{f_{2}} \approx m_{a_{2}}$.

From the parton model considerations 29 and the QCD analysis of hard exclusive processes [30, 31] it follows that for large $Q^{2}$ the tensor meson production amplitude with zero helicity (in the $\gamma^{*} \gamma$ center-of-mass system) should tend to the constant value (with logarithmic accuracy), and other amplitudes should be suppressed by powers of $Q^{2}$. This implies that $F_{T}\left(Q^{2}\right) \sim 1 / Q^{4}$ for large $Q^{2}$. In the generalized vector meson dominance model (GVDM) such an asymptotic behavior is provided by the compensation in $Q^{2}$-channel of the contributions of ground and excited states of vector mesons $V=\rho, \omega, \phi$, $V^{\prime}=\rho^{\prime}, \omega^{\prime}, \phi^{\prime}, V^{\prime \prime}=\rho^{\prime \prime}, \omega^{\prime \prime}, \phi^{\prime \prime}$, etc. [28].

It is interesting to find out, at least roughly, how fast the angular distributions can vary with $Q^{2}$ in the reactions $\gamma^{*} \gamma \rightarrow f_{2}(1270) \rightarrow \pi \pi, \gamma^{*} \gamma \rightarrow a_{2}(1320) \rightarrow \pi^{0} \eta$, and $\gamma^{*} \gamma \rightarrow a_{2}(1320) \rightarrow \rho^{ \pm} \pi^{\mp} \rightarrow \pi^{+} \pi^{-} \pi^{0}$ for $0<Q^{2}<$ $40 \mathrm{GeV}^{2}$ (in the case of the processes $\gamma^{*} \gamma \rightarrow \pi^{0}, \eta, \eta^{\prime}$ the asymptotic regime apparently occurs near $40 \mathrm{GeV}^{2}$ ).

We put $m_{\rho}=m_{\omega}, m_{\rho^{\prime}}=m_{\omega^{\prime}}$, etc., and will consider that the resonance $f_{2}(1270)$ does not contain strange valent quarks (as $\omega, \omega^{\prime}$, etc.). Then, in GVDM, a simplest expression for $F_{T}\left(Q^{2}\right)$ with the required asymptotic behavior has the form

$$
F_{T}\left(Q^{2}\right)=\frac{1}{\left(1+Q^{2} / m_{\rho}^{2}\right)\left(1+Q^{2} / m_{\rho^{\prime}}^{2}\right)} .
$$

Figure 2 shows the $Q^{2}$ dependencies of the normalized vertex functions $2\left|V_{\lambda_{1}, \lambda_{2}}^{(\lambda)}\left(T ; m_{T}^{2}, Q^{2}\right)\right| /\left(g_{T \gamma \gamma} m_{T}^{2}\right)$ calculated according Eqs. (2) -(6) and (10) at $m_{\rho}=0.775$ $\mathrm{GeV}, m_{\rho^{\prime}}=1.465 \mathrm{GeV}$, and $s=m_{T}^{2}$. As is seen from Fig. 2, the main at $Q^{2}=0$ vertex function with helicity $\lambda=2$ decreases very rapidly with increasing $Q^{2}$. For $Q^{2} \gtrsim 10 \mathrm{GeV}^{2}$ the vertex function with helicity $\lambda=0$ be- 
comes main and close to its asymptotic value.

The angular distributions in the reactions $\gamma^{*} \gamma \rightarrow$ $f_{2}(1270) \rightarrow \pi \pi$ [26], $\gamma^{*} \gamma \rightarrow a_{2}(1320) \rightarrow \pi^{0} \eta$, and $\gamma^{*} \gamma \rightarrow a_{2}(1320) \rightarrow \rho^{ \pm} \pi^{\mp} \rightarrow \pi^{+} \pi^{-} \pi^{0}$ reshape as $Q^{2}$ increases with the same rate.

The differential cross sections for $\gamma^{*} \gamma \rightarrow f_{2}(1270) \rightarrow$ $\pi \pi$ and $\gamma^{*} \gamma \rightarrow a_{2}(1320) \rightarrow \pi^{0} \eta$ (integrated over the azimuth angle of one of the outgoing mesons in the $\gamma^{*} \gamma$ center-of-mass system) have the following form: $\sin ^{4} \theta$ for the tensor meson decays from the helicity $\lambda= \pm 2$ states, $4 \cos ^{2} \theta \sin ^{2} \theta$ for $\lambda= \pm 1$, and $\frac{2}{3}\left(3 \cos ^{2} \theta-1\right)^{2}$ for $\lambda=0$, where $\theta$ is the polar angle of one of the outgoing mesons. These angular distributions are equally normalized. Thus, the $\sin ^{4} \theta$ distribution dominating at $Q^{2}=0$ should be replaced by the $\frac{2}{3}\left(3 \cos ^{2} \theta-1\right)^{2}$ distribution with increasing $Q^{2}$.

The amplitude of the reaction $\gamma^{*} \gamma \rightarrow a_{2}(1320) \rightarrow$ $\rho^{ \pm} \pi^{\mp} \rightarrow \pi^{+} \pi^{-} \pi^{0}$ is described by two diagrams and therefore the corresponding angular distributions for $\lambda= \pm 2, \pm 1$, and 0 have a rather cumbersome form. Nevertheless these distributions are sensitive to the $a_{2}(1320)$ helicity $\lambda$. They are exhaustively represented in Refs. [21 25]. Here we consider as an example the contribution of one diagram $\gamma^{*} \gamma \rightarrow a_{2}(1320) \rightarrow \rho^{+} \pi^{-} \rightarrow \pi^{+} \pi^{-} \pi^{0}$ only. Then the angular distributions (integrated over the azimuth angle of the outgoing $\pi^{-}$meson) corresponding to the $\lambda= \pm 2, \pm 1$, and 0 helicity contributions are

$$
\begin{gathered}
\sin ^{2} \theta_{\rho^{+}} \sin ^{2} \theta_{\pi^{+}}\left(\cos ^{2} \theta_{\rho^{+}} \sin ^{2} \varphi_{\pi^{+}}+\cos ^{2} \varphi_{\pi^{+}}\right) \\
\sin ^{2} \theta_{\pi^{+}}\left[\sin ^{2} \varphi_{\pi^{+}}\left(2 \cos ^{2} \theta_{\rho^{+}}-1\right)^{2}+\cos ^{2} \varphi_{\pi^{+}} \cos ^{2} \theta_{\rho^{+}}\right] \\
6 \sin ^{2} \theta_{\rho^{+}} \sin ^{2} \theta_{\pi^{+}} \cos ^{2} \theta_{\rho^{+}} \sin ^{2} \varphi_{\pi^{+}}
\end{gathered}
$$

respectively, where $\theta_{\rho^{+}}$is the polar angle of the $\rho^{+}$in the $\gamma^{*} \gamma$ center-of-mass system, with the z-axis along the incident photon direction; the angles $\theta_{\pi^{+}}$and $\varphi_{\pi^{+}}$describe the decay of the $\rho^{+}$in its helicity system; $\varphi_{\pi^{+}}$is measured from the plane defined by the momenta of the $\rho^{+}$and photons. As $Q^{2}$ increases, the distribution from Eq. (11) should be replaced by that from Eq. (13).

Note that the form factors of a more general form than that in Eq. (10) may be required for the treatment of real data, for example,

$$
F_{T}\left(Q^{2}\right)=\frac{1+\xi Q^{2}}{\left(1+Q^{2} / m_{\rho}^{2}\right)\left(1+Q^{2} / m_{\rho^{\prime}}^{2}\right)\left(1+Q^{2} / m_{\rho^{\prime \prime}}^{2}\right)}
$$

with varying masses $m_{\rho^{\prime}}$ and $m_{\rho^{\prime \prime}}$ and an additional free parameter $\xi$.

Deviations from the above picture are possible in principle since the tensor meson production in $\gamma^{*} \gamma$ collisions can be described in the general case by three independent invariant amplitudes. However, our scenario is based on the well-established dominance of the $\lambda= \pm 2$ helicity states in the tensor meson production by two real photons. This allows us to hope that possible deviations will be small.

Thus, the experiments on the reactions $\gamma^{*} \gamma \rightarrow$ $f_{2}(1270)$ and $\gamma^{*} \gamma \rightarrow a_{2}(1320)$ will allow to check the theoretical predictions about the changing of the dominant helicity amplitude with increasing $Q^{2}$. The dynamics of this change can be tracked by analyzing the angular distributions of the final mesons in the reactions $\gamma^{*} \gamma \rightarrow f_{2}(1270) \rightarrow \pi \pi, \gamma^{*} \gamma \rightarrow a_{2}(1320) \rightarrow \pi^{0} \eta$, and $\gamma^{*}\left(Q^{2}\right) \gamma \rightarrow a_{2}(1320) \rightarrow \rho^{ \pm} \pi^{\mp} \rightarrow \pi^{+} \pi^{-} \pi^{0}$. The information obtained on three transition form factors, corresponding to the $\lambda=2,1$, and $0 \gamma^{*} \gamma$ helicity amplitudes, would be crucial for the selection of dynamical models of the $f_{2}(1270)$ and $a_{2}(1320)$ resonance production.

We have shown that the transition to the asymptotic behavior of QCD in the amplitudes $\gamma^{*}\left(Q^{2}\right) \gamma \rightarrow$ $f_{2}(1270), a_{2}(1320)$ is provided by the compensation of the contributions of ground vector states $\rho$ and $\omega$ in $Q^{2}$ channel with those of their radial excitations.

More recently, the Belle Collaboration represented the first data on the processes $\gamma^{*}\left(Q^{2}\right) \gamma \rightarrow f_{2}(1270)$ extracted from the measured differential cross section of the reaction $\gamma^{*} \gamma \rightarrow f_{2}(1270) \rightarrow \pi^{0} \pi^{0}$ for $Q^{2}$ up to $30 \mathrm{GeV}^{2}$ [32]. In Fig. 3, the curves transferred from Fig. 2 are compared with the Belle data which we multiplied by a factor $\left(1+Q^{2} / m_{f_{2}}^{2}\right)$ in order to match the definition of the transition form factors with $\lambda=2,1$, and 0 used in Ref. [32] with our definition of the normalized vertex functions. The theoretical curves are in satisfactory agreement with the data.

The present work is partially supported by the RFBR grant No. 13-02-00039 and by the Interdisciplinary project No. 102 of the Siberian Branch of RAS.
[1] K.A. Olive, K. Agashe, C. Amsler et al. (Particle Data Group), Chin. Phys. C 38, 090001 (2014).

[2] D.M. Asner, T. Barnes, J.M. Bian et al. (BESIII Collab.), Int. J. Mod. Phys. A 24, S1 (2009).

[3] T. Aushev, W. Bartel, A. Bondar et al. (Su-
perKEKB/Belle II Collab.), arXiv:1002.5012.

[4] A.J. Bevan, B. Golob, Th. Mannel et al. (BaBar and Belle Collab.), Eur. Phys. J. C 74, 3026 (2014).

[5] T. Mori, S. Uehara, Y. Watanabe et al. (Belle Collab.), Phys. Rev. D 75, 051101(R) (2007). 

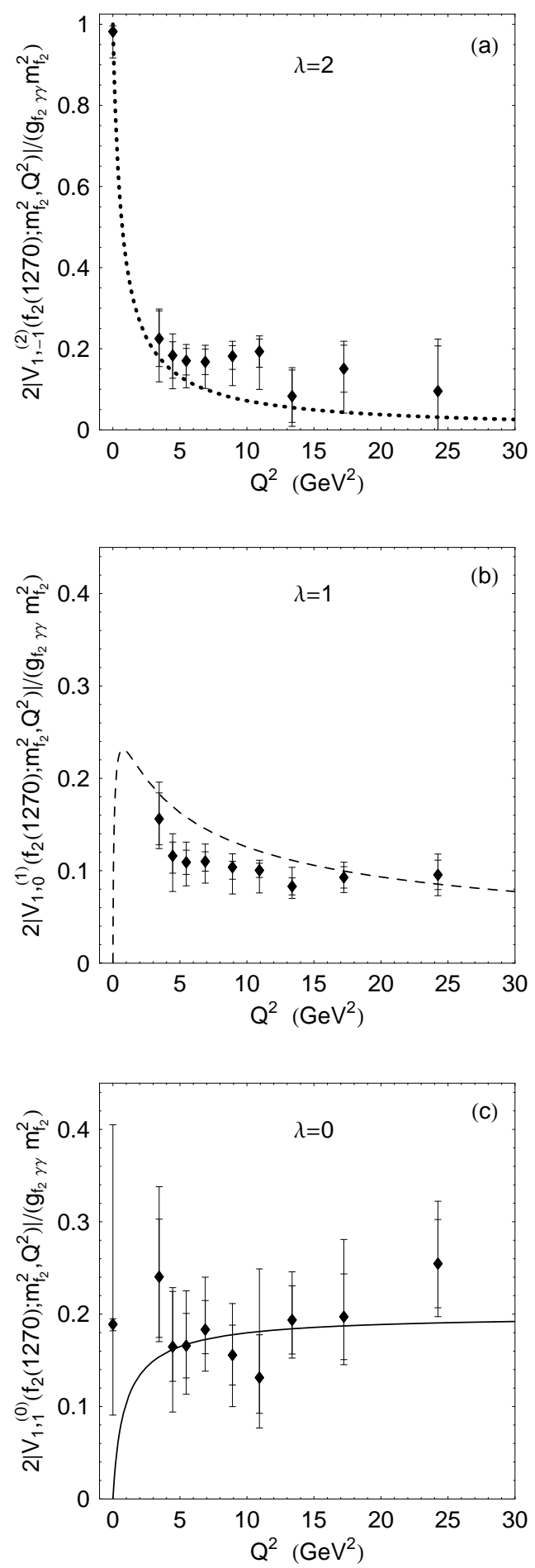

Figure 3: Comparison of the $Q^{2}$ dependencies of the normalized vertex functions $2\left|V_{\lambda_{1}, \lambda_{2}}^{(\lambda)}\left(f_{2}(1270) ; m_{f_{2}}^{2}, Q^{2}\right)\right| /\left(g_{f_{2} \gamma \gamma} m_{f_{2}}^{2}\right)$, calculated according to Eqs. (22)-(6) and (10), with the Belle data. 32]. The curves are the same as in Fig. 2. The Belle data are reduced to our normalization.
[6] T. Mori, S. Uehara, Y. Watanabe et al. (Belle Collab.), J. Phys. Soc. Jpn. 76, 074102 (2007).

[7] S. Uehara, Y. Watanabe, I. Adachi et al. (Belle Collab.), Phys. Rev. D 78, 052004 (2008).

[8] S. Uehara, Y. Watanabe, H. Nakazawa et al. (Belle Collab.), Phys. Rev. D 80, 032001 (2009).

[9] N.N. Achasov, S.A. Devyanin, and G.N. Shestakov, Phys. Lett. 108 B , 134 (1982).

[10] N.N. Achasov, S.A. Devyanin, and G.N. Shestakov, Z. Phys. C 16, 55 (1982).

[11] N.N. Achasov, G.N. Shestakov, Physics-Uspekhi 54, 799 (2011) [Usp. Fiz. Nauk 181, 827 (2011)].

[12] H.-J. Behrend, L. Criegee, T.H. Field et al. (CELLO Collab.), Z. Phys. C 49, 401 (1991).

[13] J. Gronberg, T.S. Hill, R. Kutschke et al. (CLEO Collab.), Phys. Rev. D 57, 33 (1998).

[14] B. Aubert, Y. Karyotakis, J.P. Lees et al. (BaBar Collab.), Phys. Rev. D 80, 052002 (2009).

[15] P. del Amo Sanchez, J.P. Lees, V. Poireau et al. (BaBar Collab.), Phys. Rev. D 84, 052001 (2011).

[16] S. Uehara, Y. Watanabe, H. Nakazawa et al. (Belle Collab.), Phys. Rev. D 86, 092007 (2012).

[17] J. Boyer, F. Butler, G. Gidal et al. (Mark II Collab.), Phys. Rev. D 42, 1350 (1990).

[18] H.-J. Behrend, L.Criegee, T.H. Field et al. (CELLO Collab.), Z. Phys. C 56, 381 (1992).

[19] H. Marsiske, D. Antriasyan, H.W. Bartels et al. (Crystal Ball Collab.), Phys. Rev. D 41, 3324 (1990).

[20] D. Antreasyn, D. Aschman, D. Besset et al. (Crystal Ball Collab.), Phys. Rev. D 33, 1847 (1986).

[21] Ch. Berger, A. Deuter, H. Genzel et al. (PLUTO Collab.), Phys. Lett. 149 B, 427 (1984).

[22] S.E. Baru, A.E. Blinov, V.T. Blinov et al. (MD-1 Collab.), Z. Phys. C 48, 581 (1990).

[23] H. Albreht, T. Hamacher, R.P. Hofmann et al. (ARGUS Collab.), Z. Phys. C 74, 469 (1997).

[24] M. Acciarri, O. Adriani, M. Anuilar-Btnitez et al. (L3 Collab.), Phys. Lett. B 413, 147 (1997).

[25] K. Abe, K. Abe, I. Adachi et al. (Belle Collab.), arXiv:hep-ex/0610022

[26] N.N. Achasov and V.A. Karnakov, Z. Phys. C 30, 141 (1986).

[27] N.N. Achasov, A.I. Goncharenko, A.V. Kiselev, and E.V. Rogozina, Phys. Rev. D 88, 114001 (2013); Phys. Rev. D 89, 059906(E) (2014).

[28] N.N. Achasov, A.V. Kiselev, and G.N. Shestakov, arXiv:1504.07346

[29] G. Köpp, T.F. Walsh, and P. Zerwas, Nucl. Phys. B 70, 461 (1974).

[30] V.L. Chernyak and A.R. Zhitnitsky, Phys. Rep. 112, 173 (1984).

[31] V.N. Baier and A.G. Grozin, Sov. J. Part. Nucl. 16, 1 (1985) [Fiz. Elem. Chast. At. Yad. 16, 5 (1985)].

[32] M. Masuda, S. Uehara, Y. Watanabe et al. (Belle Collab.), arXiv:1508.06757 